\title{
Qualitätsindikatoren im Kontext der Qualitåtsstrategie der Schweiz
}

Die schweizerische Regierung hat im Oktober 2009 eine Qualitätsstrategie für das Gesundheitswesen [1] gut geheißen. Damit bekräftigte sie, dass sie die im Krankenversicherungsgesetz verankerte Verpflichtung zu qualitätssichernden Maßnahmen mit erhöhter Priorität durchsetzen will und dabei beabsichtigt, die Führungsrolle zu übernehmen.

Im Mai 2011 verabschiedete die schweizerische Regierung in einem zweiten Schritt den Bericht zur Konkretisierung der Qualitätsstrategie [2]. Einerseits soll bis zum Jahr 2015 für deren Umsetzung eine neue gesetzliche Finanzierungsbasis geschaffen werden. Andererseits besteht die Absicht, ein nationales Qualitätsinstitut zu gründen, dem in weiten Bereichen die Umsetzung der Qualitätsstrategie übertragen werden soll. Bis für die beiden Projekte die entsprechenden gesetzlichen Grundlagen vom Parlament verabschiedet werden, sieht die Regierung Sofortmaßnahmen vor.

In Anlehnung an die in verschiedenen Ländern gestarteten nationalen Verbesserungsprogramme soll ein erstes Nationales Qualitätsprogramm für die Jahre 2011 bis 2014 angegangen werden. Die inhaltlichen Schwerpunkte sind:

1.) die Reduktion von Spitalinfektionen,

2.) die Verbesserung der Medikationssicherheit,

3.) die Erhöhung der Sicherheit bei invasiven

Eingriffen („safe surgery“).

Damit wurde dem Thema Patientensicherheit in den Spitälern erste Priorität eingeräumt. Weiter plant das Bundesamt für Gesundheit die Publikation der Qualitätsindikatoren (Mortalitäts- und Fallzahlen; auf der Basis der G-IQI) [3] ab Ende 2011 in die Breite zu fahren, indem die Daten für alle akutsomatischen Kliniken der Schweiz neu publiziert werden. Es ist erfreulich, dass im Rahmen der Initiative Qualitätsmedizin (IQ $\left.{ }^{\mathrm{M}}\right)$ ein offenes Benchmarking auch mit den großen Universitätsklinken Deutschlands möglich wird. Damit konnte auch die Akzeptanz des gewählten Indikatorenmodells erhöht werden. Ein weiteres Projekt im Rahmen der Sofortmaßnahmen wird schließlich die Wahl von Qualitätsindikatoren für den ambulanten ärztlichen Bereich sein.

Autorenerklärung: Der Autor erklärt, dass keine relevanten finanziellen Verbindungen in Bezug auf dieses Manuskript bestehen.
Literatur

1 http://www.swiss-q.admin.ch/pdf/Qualitaetsstrategie.pdf

2 http://www.swiss-q.admin.ch/pdf/ 110525_Bericht_D.pdf

3 http://www.news.admin.ch/dokumentation/ 00002 $/ 00015 /$ ?lang $=$ de\&msg-id $=34667$

\section{Langenegger}

Qualitätsmanagement

Schlüsselwörter Qualitätsstrategie Patientensicherheit $\checkmark$ Qualitätsindikatoren

Keywords quality strategy $\checkmark$ patient safety quality indicators
Institut

Bundesamt für Gesundheit, Bern, Schweiz

Bibliografie

DOI 10.1055/s-0031-1286074

Dtsch Med Wochenschr 2011 . 136: S45 - (c) Georg Thieme Verlag KG Stuttgart · New York . ISSN 0012-0472

Korrespondenz Manfred Langenegger, lic.rer.pol.

Projektleiter Qualitätssicherung Bundesamt für Gesundheit Schwarzenburgstrasse 165 CH-3003 Bern, Schweiz Tel. +41313229042 eMail manfred.langenegger@ bag.admin.ch 